\title{
Curcumin attenuates colistin-induced peripheral neurotoxicity in mice
}

Chongshan Dai ${ }^{1}$, Xilong Xiao', Yuan Zhang ${ }^{1}$, Biao Xiang ${ }^{1}$, Daniel Hoyer ${ }^{2,3,4}$, Jianzhong Shen ${ }^{1}$, Tony Velkov² ${ }^{*}$, Shusheng Tang ${ }^{1 *}$

${ }^{1}$ College of Veterinary Medicine, China Agricultural University, Beijing 100193, P. R.

China.

2.Department of Pharmacology \& Therapeutics, School of Biomedical Sciences, Faculty of Medicine, Dentistry and Health Sciences, The University of Melbourne, Parkville, Victoria, 3010, Australia.

${ }^{3}$ The Florey Institute of Neuroscience and Mental Health, The University of Melbourne, 30 Royal Parade, Parkville, VIC, 3052, Australia.

${ }^{4}$ Department of Molecular Medicine, The Scripps Research Institute, 10550 N. Torrey Pines Road, La Jolla, CA 92037, USA.

Running Title: Curcumin attenuates colistin peripheral neurotoxicity

Co-correspondence:

Shusheng Tang, +86 6273 3377. E-mail: tssfj@cau.edu.cn OR

Tony Velkov, Telephone: +61 38344 9846. E-mail: Tony.Velkov@unimelb.edu.au 


\section{Suppl. Material and methods}

\section{Instrumental conditions and sample preparation for colistin and curcumin determination}

\section{Instrumental conditions}

Chromatographic analyses were performed on an Acquity ultraperformance liquid chromatography system (Waters, Milford, MA,USA) and separations were achieved on an Acquity BEH C18 column(50 mm × $2.1 \mathrm{~mm}$ i.d., $1.7 \mu \mathrm{m}$ particle size) (Waters, Milford, MA, USA)with column oven temperature of $30^{\circ} \mathrm{C}$. With an injection volume of $5 \mu \mathrm{L}$, the analyses were separated with mobile phases comprised of $0.5 \%$ formic acid in water (solvent $\mathrm{A}$ ) and $0.5 \%$ formic acid in acetonitrile (solvent $B$ ). The flow rate was $0.3 \mathrm{~mL} / \mathrm{min}$ with the following gradient program: $0-0.15 \mathrm{~min}, 95 \% \mathrm{~A} ; 0.15-0.35 \mathrm{~min}, 95-30 \% \mathrm{~A} ; 0.35-$ 0.5min, 30\%A; 0.5-0.8 min, 30-5\% A; 0.8-2.5min, 5\%A; 2.5-4 min, 5-95\% A. The UHPLC system was coupled to a Micromass Xevo Quattro-LC triple quadrupole mass spectrometer (Waters, Milford, MA, USA), which was operated with an electrospray ionization (ESI) source in positive mode as the following parameters: cone voltage, $35 \mathrm{~V}$; capillary voltage, $2.5 \mathrm{kV}$; source temperature, $150{ }^{\circ} \mathrm{C}$; desolvation temperature, $500{ }^{\circ} \mathrm{C}$; cone gas (N2) flflow rate, $50 \mathrm{~L} / \mathrm{h}$; desolvation gas (N2) flflow rate, $800 \mathrm{~L} / \mathrm{h}$; collision gas flow, $0.15 \mathrm{~mL} / \mathrm{min}$

Multiple reaction monitoring (MRM) was performed and the corresponding transitions and collision energy were as follows: Curcumin, m/z 369.6 > 177.01 (collision energy, $21 \mathrm{eV}$; transition for quantification), m/z 369.6 > 145 (collision energy, $28 \mathrm{eV}$ ). Colistin A, m/z 391 $>385$ (collision energy, $8 \mathrm{eV}$; transition for quantification), $\mathrm{m} / \mathrm{z} 391>101$ (collision energy, $15 \mathrm{eV}$ ); colistin $\mathrm{B}, \mathrm{m} / \mathrm{z} 386>380$ (collision energy, $8 \mathrm{eV}$; transition for quantification), $\mathrm{m} / \mathrm{z}$ $386>101$ (collision energy, $15 \mathrm{eV}$ ). Auto dwell time was applied to ensure that approximately 15 data points were acquired for each chromatographic peak. Data acquisition was performed using Mass Lynx software v4.1 with the QuanLynx program (Waters, Milford, MA, USA).

\section{Sample preparation}

Representative portions of $0.1 \mathrm{~g}$ homogenized samples were weighed and transferred to $50 \mathrm{~mL}$ capped polypropylene centrifuge tubes for preparation of routine testing samples. 
Meanwhile, appropriate amount of working solutions were added to blank samples for the preparation of fortified samples. For colistin sample extraction, $10 \mathrm{~mL}$ of extractant constituted of methanol: water $(70: 30, \mathrm{v} / \mathrm{v})$ was added to each tube, and then the mixtures were vortexed for $5 \mathrm{~min}$, followed by centrifugation at $9391 \mathrm{~g}$ for $10 \mathrm{~min}$ at $4{ }^{\circ} \mathrm{C}$. The supernatant was collected. The extract was added to $15 \mathrm{ml}$ with a volumetric flask and followed by centrifugation at $9391 \mathrm{~g}$ for $10 \mathrm{~min}$ at $4{ }^{\circ} \mathrm{C}$. Finally evaporated to less than 1 $\mathrm{mL}$ under nitrogen at $50{ }^{\circ} \mathrm{C}$. For curcumin sample extraction, $10 \mathrm{~mL}$ of extractant constituted of $10 \%$ trichloroacetic acid: acetonitrile (40:60, v/v) was added to each tube, and then the mixtures were vortexed for $5 \mathrm{~min}$, followed by centrifugation at $9391 \mathrm{~g}$ for 10 min at $4{ }^{\circ} \mathrm{C}$. The supernatant was collected. The $\mathrm{pH}$ of combined extraction solution was adjusted to 9.0 and centrifuged at $9391 \mathrm{~g}$ for $10 \mathrm{~min}$ at $2{ }^{\circ} \mathrm{C}$. The supernatant was transferred onto the Oasis WCX cartridge, which was preconditioned with $3 \mathrm{~mL}$ of methanol. The cartridge was rinsed with $3 \mathrm{~mL}$ of water, $3 \mathrm{~mL}$ of methanol and $3 \mathrm{~mL}$ of $5 \%$ formic acid in water. The analytes were eluted with $3 \mathrm{~mL}$ of eluent constituted of formic acid: water: methanol (30: 10: 60, v/v) and evaporated to less than $1 \mathrm{~mL}$ under nitrogen at $50{ }^{\circ} \mathrm{C}$. The extract was reconstituted to $1 \mathrm{~mL}$ with water, vortexed for $1 \mathrm{~min}$, and centrifuged at $14,024 \mathrm{~g}$ for $20 \mathrm{~min}$ at $2{ }^{\circ} \mathrm{C}$. The supernatant was filtered through a $0.2 \mu \mathrm{m}$ syringe filter prior to instrumental analysis. 
Table S1 Primer sequences used for quantitative real-time PCR

\begin{tabular}{|c|c|c|}
\hline Gene & Direction & Primer sequence ( $5^{\prime}$ to $3^{\prime}$ ) \\
\hline \multirow{2}{*}{ NGF } & forward & 5'-AGG CCC ATG GTA CAA TCC CTT TCA-3' \\
\hline & reverse & 5'-ATC TCC AAC CCA CAC ACT GAC ACT-3' \\
\hline \multirow[t]{2}{*}{ Nrf2 } & forward & 5'-CAC ATT CCC AAA CAA GAT GC-3' \\
\hline & reverse & 5'-TCT TTT TCC AGC GAG GAG AT-3' \\
\hline \multirow[t]{2}{*}{$\mathrm{HO}-1$} & forward & 5'-CGT GCT CGA ATG AAC ACT CT-3' \\
\hline & reverse & 5'-GGA AGC TGA GAG TGA GGA CC-3' \\
\hline \multirow[t]{2}{*}{ Caspase-9 } & forward & 5'-TGC ACT TCC TCT CAA GGC AGG ACC-3 \\
\hline & reverse & 5'-TCC AAG GTC TCC ATG TAC CAG GAG C-3' \\
\hline \multirow[t]{2}{*}{ Bax } & forward & 5'-TTC ATC CAG GAT CGA GCA GG-3' \\
\hline & reverse & 5'-TCC GTG TCC ACG TCA GCA AT-3' \\
\hline \multirow[t]{2}{*}{ Caspase-3 } & Forward & 5'- TCG CAG CAT TTC TCC TAA G -3' \\
\hline & Reverse & 5'- CAA CAA AGC CAG TCT AAA C -3' \\
\hline \multirow[t]{2}{*}{ Akt } & Forward & 5'-CGA CGT AGC CAT TGT GAA GG-3' \\
\hline & Reverse & 5'-CTT GGC CGC TCT GTC TTC AT -3' \\
\hline \multirow[t]{2}{*}{ mTOR } & Forward & 5'-TAC GTC ACC ATG GAG CTT CG -3' \\
\hline & Reverse & 5'-GAC AAC TGG ATC GCT TGA GG -3' \\
\hline \multirow[t]{2}{*}{ GAPDH } & Forward & 5'-ACA GTC CAT GCC ATC ACT GCC-3' \\
\hline & reverse & 5'-GCC TGC TTC ACC ACC TTC TTG-3' \\
\hline
\end{tabular}


Figure S1 The purity analysis of curcumin

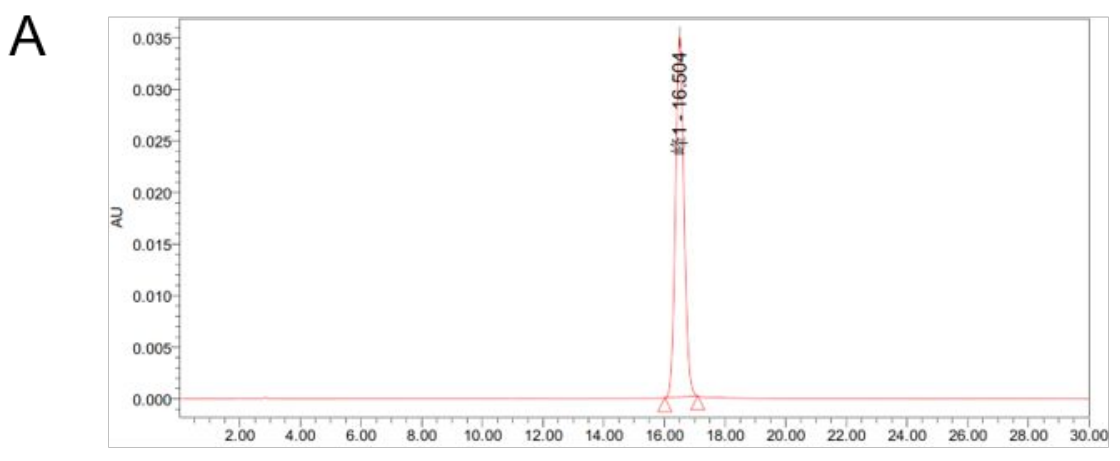

B

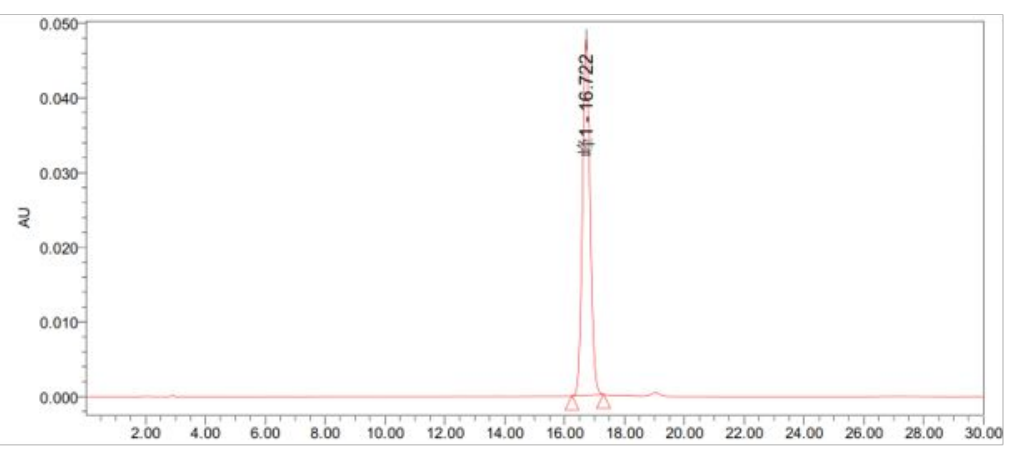

C

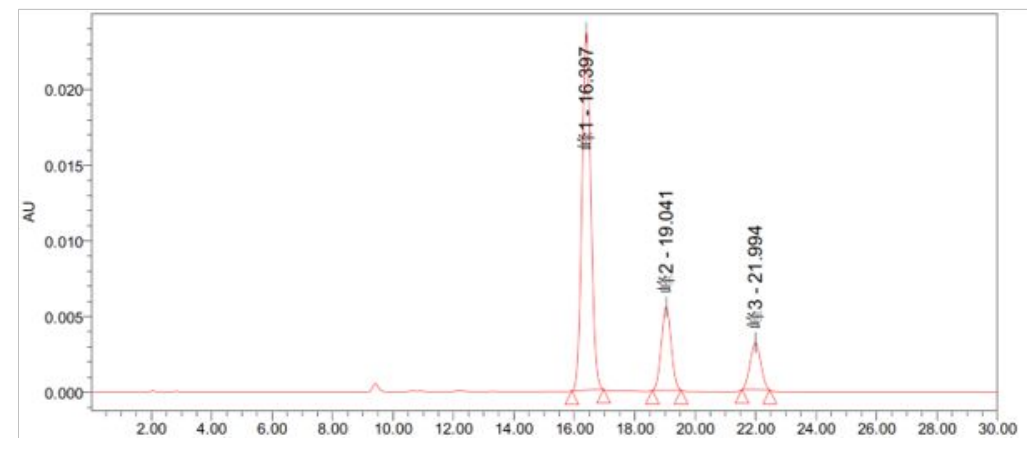

A, Curcumin (purity $\geq 99.5 \%$; Sigma); B, Curcumin, (purity $\geq 98 \%$; Aladdin Reagent Co., Ltd); C, Curcumin (purity $\geq 65 \%$; Aladdin Reagent Co., Ltd). The purity of the curcumin (i.e., curcumin, labeled purity is $\geq 98 \%$ from Aladdin Reagent Co., Ltd) used in our animal experiments is $\sim 98.3 \%$. 